\title{
Pneumatosis intestinalis in a patient with trichobezoar - Rare association
}

\author{
Varuna B. Pathirana ${ }^{\mathrm{a},{ }^{*}}$, Rakitha V. Paranamanna ${ }^{\mathrm{a}}$, Gayan R. M. Rathnayaka ${ }^{\mathrm{a}}$, Mahanama. Gunasekara ${ }^{\mathrm{a}}$ \\ ${ }^{a}$ Department of General Surgery, National Hospital, Colombo, Sri Lanka.
}

\begin{abstract}
This case report describes a young girl who presented with chronic epigastric pain and abdominal mass without noticeable psychiatric illness or trichotillomania and subsequently trichobezoar was found to be the reason for her symptoms. She underwent laparotomy to retrieve the bezoar. During laparotomy extensive pneumatosis of the small bowel was noted where this association was not previously reported in the literature. Since pneumatosis was not symptomatic no bowel resection was carried out. She made an uneventful recovery. This illustrates that trichobezoar is an important cause to consider in young females with chronic abdominal pain even in the absence of clear evidence for trichotillomania. Rarely, this can be associated with intestinal pneumatosis. Intestinal pneumatosis does not warrant treatment unless it causes symptoms.

Keywords: Trichobezoar; epigastric pain; pneumatosis intestinalis; trichotillomania; pneumoperitoneum
\end{abstract}

\section{INTRODUCTION}

Trichobezoar is a mass of ingested hair within the gastrointestinal tract ${ }^{[1]}$. This rare cause was found to be the reason for chronic epigastric pain of a young girl in this case report without apparent psychiatric illness. She was incidentally found to have pneumatosis intestinalis (PI) which may be or may not be related to trichobezoar $^{[2]}$.

\section{CASE REPORT}

A 17-year-old girl presented with epigastric pain for one-year duration associated with nausea, postprandial abdominal fullness, early satiety, and episodic vomiting occurring 3-4 times a month. Her bowel habits were normal and there was no per rectal bleeding or melena. She was admitted to the hospital due to worsening of symptoms over two weeks with more frequent vomiting. She denies trichotillomania and there was no

\footnotetext{
* Corresponding author: Varuna Bhagya Pathirana Mailing address: Department of General Surgery, Ward 32, National Hospital, Colombo, Sri Lanka.

E-mail: varunapath@gmail.com

Received: 29 December 2020 / Accepted: 28 January 2021
}

history of psychiatric illness or behavioral abnormality and her school performances were average.

She was not pale, anicteric, BMI was $17.18 \mathrm{~kg} / \mathrm{m}^{2}$ and no abnormality was noted in her hair. Abdominal examination revealed a firm lump involving epigastric and right hypochondria extending $6 \mathrm{~cm}$ from the costal margin. The rest of the abdominal examination was unremarkable. Her hemoglobin was $12.7 \mathrm{~g} / \mathrm{dl}$, serum albumin was $4.0 \mathrm{~g} / \mathrm{dl}$ and the rest of the laboratory investigations, including bilirubin levels, serum amylase, and serum electrolytes were within the normal range. Gastroduodenoscopy revealed large trichobezoar where the scope was not negotiable beyond the body of the stomach (Figure 1). CECT showed grossly distended stomach with non-enhancing intraluminal mass extending up to the first part of duodenum suggestive of a bezoar (Figure 2) and intramural gas in the small and large intestine suggestive of pneumatosis.

She underwent laparotomy and the bezoar was retrieved via gastrotomy (Figure 3). A gastric wall thickening was observed with a thickness of 5-6 mm. Distal jejunum and entire ileum showed gaseous outpouchings of variable size ranging from few millimeters to $1 \mathrm{~cm}$ indicating extensive intestinal Pneumatosis (Figure 4) and was left unattended since the patient was asymptomatic. She made the uneventful postoperative recovery. 

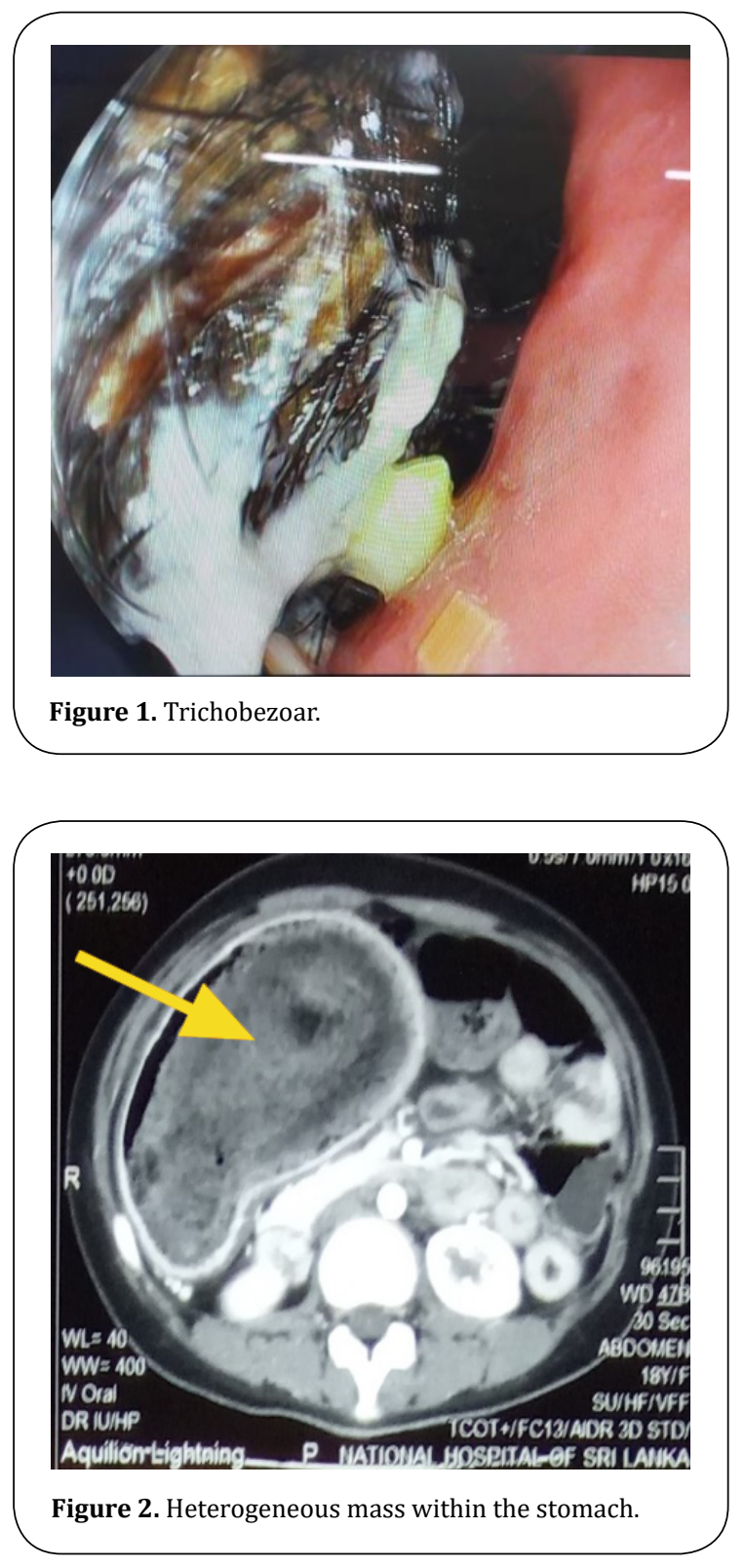

She was referred to a psychiatrist on discharge from the hospital and no active treatment was recommended.

\section{DISCUSSION}

Bezoars are masses formed from ingested foreign material and identified in less than $5 \%$ of all upper gastrointestinal endoscopy ${ }^{[1]}$. They are most commonly found in the stomach. Trichobezoar is the rarest of them accounting for $6 \%$ of all bezoars and invariably related to trichotillomania and trichophagia ${ }^{[1]}$ which can be a part of a psychiatric illness. Even though the presence of trichobezoar is a piece of clear evidence for trichophagia, some patients may deny these habits similar to this case.

Bezoars can be asymptomatic particularly when they are small and symptomatic patients have epigastric pain, nausea, and vomiting, early satiety which is nonspecific and can be overlooked as young patients may not undergo routine endoscopy leading to delay of diagnosis. Because around $90 \%$ of these patients are young females ${ }^{[1,4]}$, this case illustrates the importance of considering trichobezoar as a cause for unexplained abdominal pain in a young female even with the absence of clear psychiatric illness or evidence for trichotillomania.

Trichobezoar needs early intervention since it is known for many complications such as gastric erosion and bleeding, perforation, and gastric outlet obstruction. The extension of trichobezoar into the small bowel which is known as Rapunzel Syndrome can lead to small bowel obstruction, pancreatitis, and cholangitis ${ }^{[1,3,5]}$. Open surgery is considered the best method since it is technically easy and has a high success rate of complete removal of the bezoar with low complication rates ${ }^{[1,3,5]}$. Laparoscopic and endoscopic interventions are shown to be less effective ${ }^{[1,3,5]}$. Most patients will only need gastrotomy and/or enterotomy for retrieval of bezoar, but complicated cases may require subtotal gastrectomy or intestinal resection ${ }^{[1]}$. Following the successful removal of trichobezoar patients will need surveillance endoscopy for few years to confirm the patient has come out of trichotillomania ${ }^{[3]}$.

Pneumatosis intestinalis (PI) is a rare condition that has a wide range of clinical presentations from benign asymptomatic condition to a life-threatening surgical emergency due to adhesion obstruction or spontaneous bowel perforation ${ }^{[2,6]}$. Morphologically PI is seen in "bubbles like" type (cystoides) which was noted in this patient or a continuous band-like type ${ }^{[2]}$. It may be idiopathic (primary), occasionally identified in asymptomatic patients, or secondary to various gastrointestinal or pulmonary diseases ${ }^{[2,7]}$. Inflammatory bowel disease, chronic bowel infections and infestations, bowel obstruction and pyloric obstruction, diverticulitis, bowel ischemia, toxic megacolon are some of the common gastrointestinal conditions related to $\mathrm{PI}^{[7,8]}$. Some of the non-gastrointestinal conditions related to PI are pulmonary diseases like asthma, and emphysema, collagen vascular diseases, immunosuppression, organ transplantation ect ${ }^{[7,8]}$. Direct association with trichobezoar and PI is not documented in the literature. Chronic inflammation of the bowel wall secondary to hair, partial pyloric, and small bowel obstruction may have contributed to pneumatosis in this patient since these causes are known to associate with PI ${ }^{[7]}$. Most of the time PI will present with symptoms related 


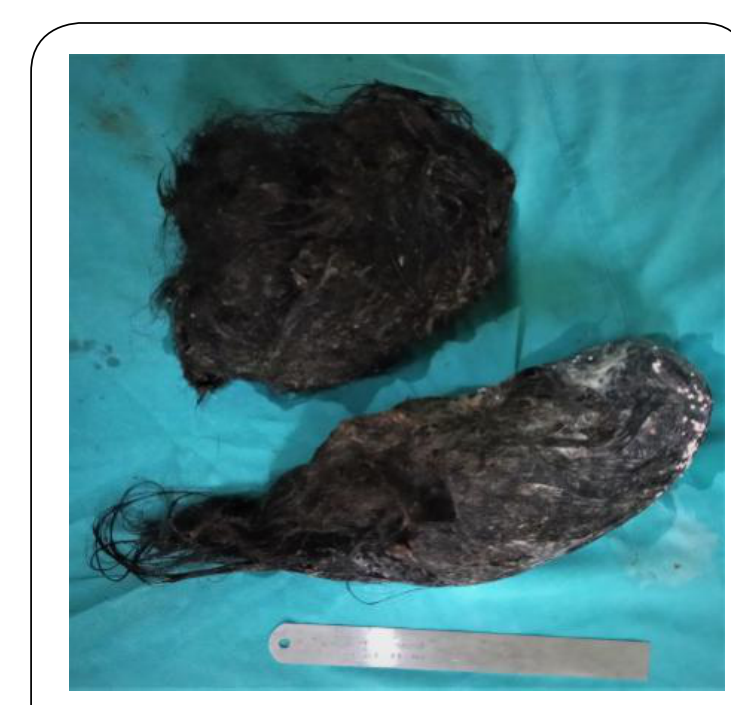

Figure 3. Retrieved trichobezoar.

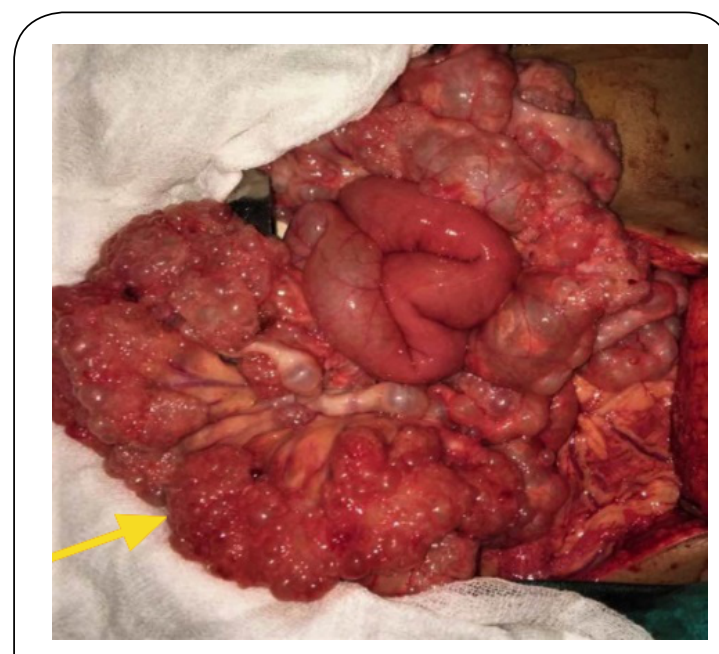

Figure 4. Distal jejunum and Ileum showing Pneumatosis intestinalis.

to its underlying disease. However, vomiting, abdominal distention, weight loss with the involvement of the small bowel and diarrhea, hematochezia with the involvement of the large bowel is reported as the symptoms related to $\mathrm{PI}^{[8]}$.

A plain abdominal x-ray may reveal pneumatosis but a contrast-enhanced CT scan of the abdomen is more sensitive in detecting PI and particularly in acute presentation it can help in identifying Intraluminal gas from intramural gas, the air within the biliary system, ischemic bowel, pneumoperitoneum, ascites which will help in the decision to ope rate ${ }^{[2,6,7]}$.

Pneumatosis intestinalis is treated according to the underlying cause and surgery is usually reserved for patients presenting with acute abdomen due to perfo- ration or obstruction. Patients with clinical and laboratory evidence of abdominal sepsis elevated serum amylase, and presence of portal venous gas need urgent surgery where asymptomatic cases are managed non operatively ${ }^{[2,6,8]}$. Pneumoperitoneum alone should not be considered as an indication for surgery since it was noted in $50 \%$ of patients with PI ${ }^{[6]}$. In this patient resection of the involved segment was not necessary as it is uncomplicated and neither feasible considering the extensive involvement of the small bowel.

\section{CONCLUSION}

Trichobezoar can be a cause for chronic unexplained abdominal pain in young females, where it may be not suspected in the absence of trichotillomania and hair loss. Trichobezoar and intestinal pneumatosis are rare clinical entities where this association was not documented previously. Pneumatosis intestinalis needs individualized treatment based on clinical presentation.

\section{DECLARATIONS}

\section{Authors' contributions}

All authors made substantial contributions to merit inclusion as co-authors. All authors approved the final manuscript.

\section{Conflict of interest}

All authors declared that there are no conflicts of interest.

\section{Ethics approval}

Not applicable.

\section{Consent for publication}

Patient consent was obtained for publication without personal details.

\section{REFERENCES}

1. García-Ramírez, B. E., Nuño-Guzmán, C. M., Zaragoza-Carrillo, R. E., Salado-Rentería, H., Gómez-Abarca, A., \& Corona, J. L. (2018). Small-bowel obstruction secondary to ileal trichobezoar in a patient with Rapunzel syndrome. Case reports in gastroenterology, 12(3), 559-565.

2. Berritto, D., Crincoli, R., Iacobellis, F., Iasiello, F., Pizza, N. L., Lassandro, F., ... \& Grassi, R. (2014). Primary pneumatosis intestinalis of small bowel: a case of a rare disease. Case reports in surgery, 2014.

3. Wolski, M., Gawłowska-Sawosz, M., Gogolewski, M., Wolańczyk, T., Albrecht, P., \& Kamiński, A. (2016). Trichotillomania, trichophagia, trichobezoar-summary of three cases. Endoscopic follow up scheme in trichotillomania. 
Psychiatr Pol, 50(1), 145-52.

4. Dindyal, S., Bhuva, N. J., Ramdass, M. J., \& Narayansingh, V. (2008). Trichobezoar presenting with the'comma sign'in Rapunzel Syndrome: a case report and literature review. Cases journal, 1(1), 1-4.

5. Gorter, R. R., Kneepkens, C. M. F., Mattens, E. C. J. L., Aronson, D. C., \& Heij, H. A. (2010). Management of trichobezoar: case report and literature review. Pediatric surgery international, 26(5), 457-463.

6. Tahiri, M., Levy, J., Alzaid, S., \& Anderson, D. (2015). An approach to pneumatosis intestinalis: Factors affecting your management. International journal of surgery case reports, 6, 133-137.

7. Ho, L. M., Paulson, E. K., \& Thompson, W. M. (2007). Pneumatosis intestinalis in the adult: benign to life-threatening causes. American Journal of Roentgenology, 188(6), 1604-1613.

8. Zhang, H., Jun, S. L., \& Brennan, T. V. (2012). Pneumatosis intestinalis: not always a surgical indication. Case reports in surgery, 2012. 